\title{
Discrimination of Sonographically Detected Breast Masses Using Frequency Shift Color Doppler Imaging in Combination With Age and Gray Scale Criteria
}

\author{
Pamela T. Bhatti, MSEE, Gerald L. LeCarpentier, PhD, \\ Marilyn A. Roubidoux, MD, J. Brian Fowlkes, PhD, \\ Mark A. Helvie, MD, Paul L. Carson, PhD
}

Abbreviations

$\mathrm{Az}$, area under the receiver operating characteristic curve; GS, gray scale; ROC, receiver operating characteristic; $\mathrm{ROI}$, region of interest; ROR, radiologist's region of interest; SWD, speedweighted pixel density; 3D, three-dimensional

Received June 15, 2000, from the Department of Radiology, University of Michigan, Ann Arbor, Michigan. Revision requested August 2, 2000. Revised manuscript accepted for publication November 14, 2000.

We are indebted to Nancy Thorson, RT, for assistance with subject recruitment, records, and procedures. Patient data collection was performed with the assistance of Jochen Krücker and Theresa Tuthill, PhD, who also contributed to statistical data analysis. Aaron Moskalik is acknowledged for software contribution. This work was supported in part by US Public Health Service grant 1R01CA55076 from the National Cancer Institute and to a lesser extent by the US Army Medical Research and Material Command under contract DAMD17-96-C-6061.

Address correspondence and reprint requests to Gerald L. LeCarpentier, PhD, University of Michigan, Department of Radiology, 200 Zina Pitcher Pl, Room 3315, Ann Arbor, MI 48109-0553.
Frequency shift color Doppler imaging was assessed in conjunction with patient age and gray scale (GS) features for discriminating benign from malignant breast masses. Thirty-eight women with sonographically detected masses scheduled for biopsy were evaluated using a 6- to 13- $\mathrm{MHz}$ scan head, and the masses were delineated in ultrasonographic image volumes. Vascularity in and around each mass was quantified using speed-weighted pixel density (SWD). Gray scale features were ranked visually on a linear scale. Combinations of indices were compared with histologic findings (18 benign and 20 malignant). Receiver operating characteristic analysis ranked performance in decreasing order from the SWD-Age-GS index, to SWD-GS, SWD-Age, Age-GS, GS criteria, SWD, and Age. At $100 \%$ sensitivity, SWDAge-GS, SWD-GS, and SWD-Age discriminated benign from malignant masses with specificities of $94 \%, 89 \%$, and $72 \%$, respectively. These results indicate significant improvement in ultrasonographic discrimination of sonographically detected breast masses by combining the vascularity measure SWD with age and GS criteria. Key words: Doppler ultrasonography; image processing; three-dimensional imaging; breast cancer; vascularity; angiogenesis.

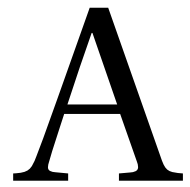

lthough their benefits far outweigh the risk of modest radiation exposure, mammograms do present some shortcomings. Screening mammograms have sensitivities of $68 \%$ to $85 \%^{1}$ and $32 \%$ specificity, ${ }^{2}$ resulting in a number of unnecessary biopsies on benign masses. Development of cost-effective, noninvasive adjunctive techniques to mammography for differentiating benign from malignant masses would significantly reduce the number of biopsies yielding negative results.

Capitalizing on the high quality of gray scale (GS) images produced by current ultrasonographic equipment, researchers have investigated discrimination techniques based on GS measures. Results describing visual 
GS criteria with a negative predictive value as high as $99.5 \%$ have been reported. ${ }^{3}$ Using the ability to visualize the speed and direction of blood flow, a host of studies have examined color flow Doppler imaging as a discrimination technique. Studies based on color flow Doppler imaging compared with those based on GS imaging yielded mixed results, leaving the role of color flow Doppler imaging still in debate. ${ }^{4}$ Pursuing a multivariable approach, by combining GS criteria with color flow and power mode Doppler, Carson et $\mathrm{al}^{5}$ reported the results of a technique that discriminates breast masses with greater success than GS or vascularity alone.

The research described here examines the performance of color flow Doppler signals, quantified as a speed-weighted pixel density (SWD), when differentiating benign from malignant breast masses. In addition, a multivariable technique is assessed using vascularity (SWD) combined with each of the following: (1) patient age, (2) GS measures, and (3) patient age and GS measures.

\section{Materials and Methods}

Volunteer patients were recruited from a group of women who had a sonographically detectable breast mass and were scheduled for biopsy. All patients were informed of the institutionally reviewed and approved experimental procedure and provided written consent before the examination. Final diagnoses were obtained by histologic analysis of biopsy specimens. A total of 43 patients were scanned. Five patients were excluded from analysis; they either had had lumpectomy or had scans that were not processed successfully or scans that showed severe Doppler artifacts due to chest wall motion during breathing.

An ultrasonographic evaluation of each patient was performed with a GE Logiq 700 scanner (GE Medical Systems, Waukesha, WI) using an M12 linear matrix array scan head (6$\mathrm{MHz}$ Doppler and 9-MHz GS). The scanned breast region measured approximately $3 \mathrm{~cm}$ long by $3.8 \mathrm{~cm}$ wide by $4 \mathrm{~cm}$ deep. The patient's electrocardiogram was acquired via a computer interface to a clinical electrocardiographic monitor and was used to trigger the footswitch of the GE scanner. Thus, cardiac gating was effectively implemented to effect image capture during systole and to maximize the Doppler sig- nal. Using the GS mode, the sonographer centered the mass in the scan window and verified the limits of the scan. Standard scanner settings are listed in Table 1. Each three-dimensional (3D) scan was acquired with a hand-controlled scanning apparatus ${ }^{6,7}$ interfaced to a personal computer, where scan head positions were acquired and stored for each image. Sixty to 90 images per scan were stored in the cine loop and then were transferred to a workstation. The scan slices were stacked to render a 3D volume. By averaging the slice-to-slice position data captured by the computer, estimated slice spacing was available for the $3 \mathrm{D}$ volume reconstruction. For a more precise morphometric analysis and multiscan registration, see the article by Krücker et al. ${ }^{8}$

Each 3D volume was displayed in AVS/Express data visualization software (Advanced Visual Systems, Waltham, MA) as a series of 3 intersecting planes (Fig. 1). In-house tools designed with AVS/Express allowed a radiologist to review the slices and determine the margins of the mass. On each overall reconstructed color flow volume, the radiologist selected a region of interest (ROI), which served to delineate the mass from the surrounding tissue, and approximated the region with a dynamically positioned and shaped ellipsoidal volume in the $3 \mathrm{D}$ volume. This ROI was referred to as the radiologist's ROI (ROR).

On the basis of the ROR, 4 regions were designated from which vascularity was measured (Fig. 2). These regions were: (1) the entire ROR, (2) the upper (proximal) half of the ROR, (3) a 3$\mathrm{mm}$ shell in the $\mathrm{x}, \mathrm{y}$, and $\mathrm{z}$ directions surrounding the ROR, and (4) the upper (proximal)-half 3-mm shell.

Within each of the 4 regions, the vascularity information was quantified as the sum of each color pixel weighted by its speed (as determined by the frequency shift color Doppler image color map), $v_{i}$, and the overall sum was normalized by the total number of pixels in the region, $N_{t}$. The result is called the SWD (where $N_{b}$ equals the total number of color pixels):

$$
\mathrm{SWD}=\sum_{i=1}^{N_{b}} \frac{v i}{N t}
$$


Table 1. Standard Scanner Settings for Patient Examination

\begin{tabular}{lr} 
GS & \\
No. of foci & 1 \\
Mean focal depth, cm & 2 \\
GS frequency, MHz & 9 \\
GS gain, dB & 22 \\
Dynamic range, dB & 69 \\
Frequency shift color Doppler imaging & \\
Gain, dB & 48 \\
Pulse repetition frequency, Hz & 1000 \\
Wall filter, Hz & 75 \\
Velocity scale, cm/s & 6 \\
\hline
\end{tabular}

Selecting the largest SWD from the 4 regions yielded the value $S W D_{\max }$. Software was developed with AVS/Express to compute SWD for all the regions.

Gray scale characteristics of the mass (margin smoothness, margin visibility, shape, height, echogenicity, attenuation, homogeneity, and "overall suggestion" of malignancy) were each ranked on a scale of 1 to 5 (low to high suggestion of malignancy) and averaged to produce $\mathrm{GS}_{\text {avg. }}{ }^{9}$ These GS criteria were similar to those used in previous studies. ${ }^{3,5}$ All GS images were observed by the same radiologist.
Vascularity, age, and GS data were initially assessed with a Bayesian discriminator, ${ }^{10}$ which was applied to each possible pair of variables to produce the combined indices SWD-Age, SWDGS, and Age-GS. For all of these calculations, $\operatorname{logSWD} \mathrm{D}_{\max }$ was used (1) to reduce the range of SWD and to avoid dominance by a few cases in the determination of discrimination functions and (2) to in some way linearize the increase in vascularity with (presumably exponential) cell growth in the cases of growing masses. The 3variable index SWD-Age-GS was also calculated from a Bayesian discriminator, this time in 3 dimensions. Including the single variables (SWD, Age, and GS), all 7 indices were compared by receiver operating characteristic (ROC) curves as well as by pair-wise univariate $z$ score tests.

\section{Results}

The results are summarized in Figures 3-6 and Tables 2-5. Of 38 lesions, 18 masses were benign (mean patient age, $48 \pm 12$ years; range, 26-70 years), and 20 were malignant (mean patient age, $56 \pm 9$ years; range, $46-72$ years). A summary of patient diagnoses is presented in Table 2, and the distribution of $\mathrm{SWD}_{\max }$ regions is provided in Table 3.

Figure 1. Three-dimensional color Doppler scan volume with ROI selected. Each slice (right) represents an original image z-plane, and approximately 90 of these are stacked to form the overall 3D reconstructed volume. The intersecting color flow planes are shown along with a blue-green 3D surface, delineating the volume for calculation of tumor vascularity. Despite the relatively smooth margins of this lesion, all multivariable indices correctly identified this metaplastic carcinoma (with foci of invasive ductal carcinoma) as malignant.
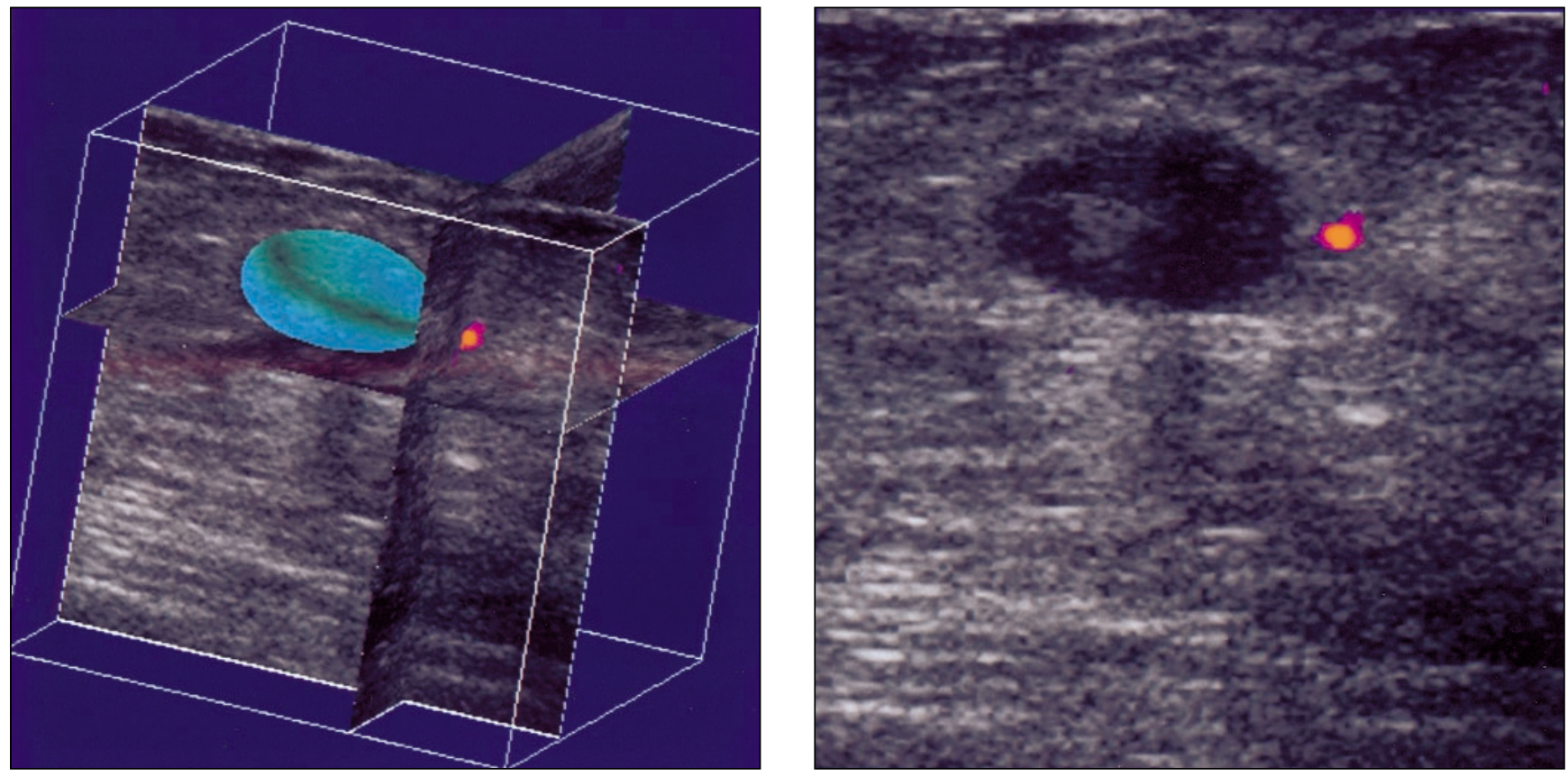


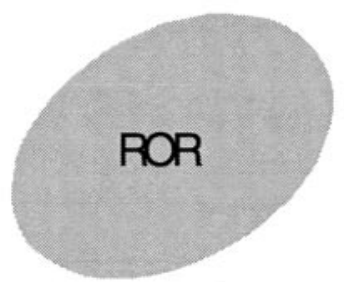

(a)

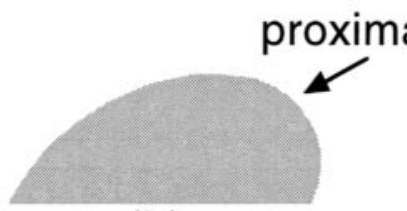

(b)

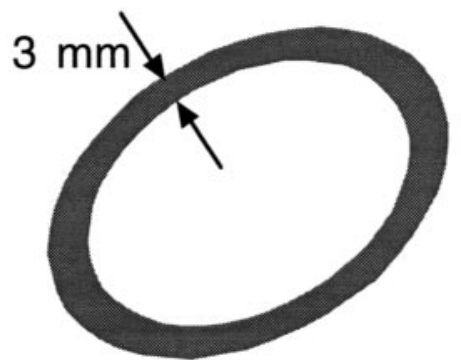

(c)

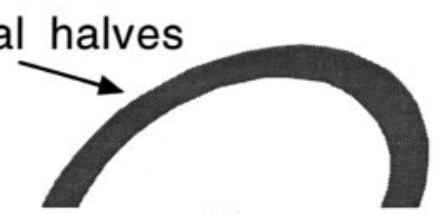

(d)

Figure 2. Schematic of z-plane cross sections of vascularity quantification regions: $\mathbf{a}$, complete ROR; $\mathbf{b}$, upper (proximal) half of the ROR; $\mathbf{c}, 3-\mathrm{mm}$ shell surrounding the ROR; $\mathbf{d}$, upper-half 3-mm shell.

To assess the various indices in relation to each other, we generated multiple two-dimensional and 3D plots similar to that presented in Figure 3. This particular example shows the relationship between SWD and patient age. We applied Bayesian decision theory ${ }^{10}$ and weighed the risk of false-negative results the same as false-positive results, and the resulting discrimination line is shown in the scatter plot of logSWD ${ }_{\text {max }}$ versus Age (Fig. 3, dark upper line). The figure illustrates the grouping of benign and malignant masses. The light lower line is drawn to illustrate discrimination at $100 \%$ sensitivity. As an alternative representation, a combined logSWD ${ }_{\text {max }}$ and Age (SWDAge) index for each case was computed using the slope of the discrimination line. Figure 4 illustrates the SWD-Age index for each case compared with diagnosis. The lines correspond to those in Figure 3, where risks are equally weighted (sensitivity, $80 \%$; specificity, $89 \%$ ) and where sensitivity is maximized to yield the lower discriminating line (sensitivity, 100\%; specificity, 72\%). Applying an analogous analysis to $\operatorname{logSWD}_{\max }$ versus $\mathrm{GS}_{\text {avg }}$ yields similar results. When $\operatorname{logSWD} \mathrm{max}_{\text {mavg }}, \mathrm{GS}_{\mathrm{avg}}$, and Age were combined, the Bayesian discriminator separated the population with $95 \%$ sensitivity and $94 \%$ specificity. When requiring a false-negative rate of 0 , this 3-variable index demonstrated $94 \%$ specificity (true-negative fraction) at $100 \%$ sen- sitivity (true-positive fraction), as shown in Figure 5. Table 4 summarizes the sensitivities and specificities when optimized for sensitivity. Figure 6 illustrates the ROC curves computed for each index. The curves depict the maximum likelihood estimate of a single binormal curve based on the respective index, ${ }^{10}$ with the area under each curve (Az) representing the diagnostic accuracy. ${ }^{11,12}$ Performance of the indices is shown. When statistical significance is disregarded, the indices that performed the best were SWDAge-GS and SWD-GS, followed by SWD-Age, Age-GS, and finally the single indices GS, SWD, and Age. Pair-wise univariate $z$ score tests of the difference among the areas under the binormal ROC curves of the all variables are shown in Table 5. Note that Age is a significantly worse diagnostic performer than all multivariable indices and that SWD-Age-GS performs statistically better than all singlevariable indices as well as Age-GS. The results also show that GS alone does not perform statistically better than SWD alone, and there is no significant difference among any of the 2-variable indices.

Figure 3. $\log S W D_{\max }$ versus Age for 38 patients studied: 18 benign (open triangles) and 20 malignant (filled triangles). A linear Bayesian discriminant analysis produced the dark upper discrimination line: $0.22=\log \left(S W D_{\max }\right)+0.478$. Age. The light lower line shows the data split with a sensitivity of $100 \%$.

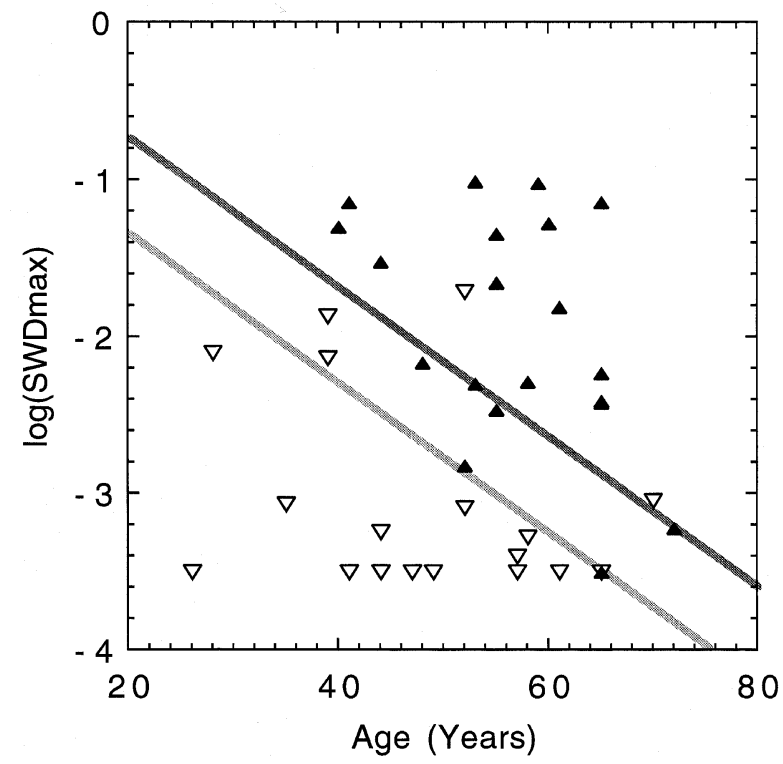




\section{Discussion}

Earlier studies assessing the performance of color flow Doppler ultrasonography in cancer discrimination have relied on varied methods for vascularity measurement. Examination of Doppler flow parameters, mean and maximum flow velocities, spectral analysis, and peak systolic and end diastolic Doppler frequency shifts have been performed. ${ }^{13-17}$ Additional investigations include (1) qualitative analysis of Doppler images, observing the amount of reflected signal associated with the mass; and (2) quantitative analysis, calculating the color pixel density or tabulating an SWD for an ROI., $5,14,18-22$

In addition to exploring flow characterization and quantification, studies have also examined the relationship among angiogenesis (suspected to be crucial for tumor growth), metastases, ${ }^{23,24}$ and color Doppler signals. ${ }^{25-27}$ By studying the correlation between color Doppler signals and tumor microvessel density, a measure of angiogenesis, researchers have shown that there is little correlation between Doppler flow parameters and microvessel density, ${ }^{25,27}$ concluding that Doppler ultrasonography yields information mainly about tumor macrovasculature.

Although these earlier studies assessing color Doppler imaging indicate mixed support for frequency shift color Doppler imaging in dis-

Figure 4. Combined SWD-Age index. The upper threshold line represents the discrimination obtained with equally weighted risks: true-positive fraction (TPF; sensitivity), 80\%; and truenegative fraction (TNF; specificity), $89 \%$. Requiring $100 \%$ sensitivity reduced specificity to $72 \%$, as shown by the lower line. Points are spread horizontally for clarity.

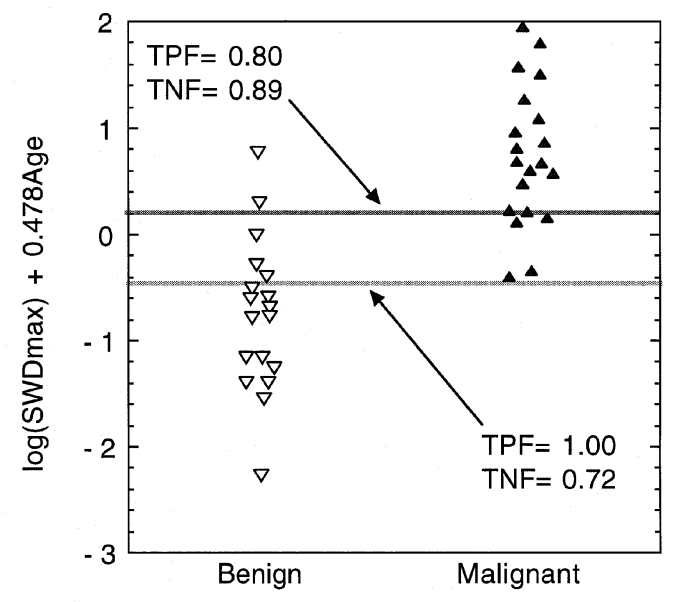

criminating benign from malignant masses, the data presented by this study indicate that $\operatorname{logSWD} \mathrm{D}_{\max }$ may be useful when applied to ultrasonographically discernible masses. Comparison between the areas under the ROC curves, that is, the diagnostic accuracy, indicated that SWD performed roughly the same as GS and in combination with Age performed better than the Age-GS index. Possibly the success of SWD may be attributed to speed weighting every pixel value. As a result, all flow information is considered when discriminating masses. The weighting of high flow speeds, thought to be illustrative of low resistance to flow often associated with vascular morphology of malignant masses, may enhance vascular quantification. In addition, highly consistent system settings presumably were critical, as was the selection of a relatively high maximum velocity $(6 \mathrm{~cm} / \mathrm{s})$, to balance sensitivity to flow with minimization of aliasing.

It has been reported that patient age may influence vascularity. There is some evidence that vascularity of malignant masses decreases with age. ${ }^{5}$ The results presented here suggest that the inclusion of patient age with SWD $(\mathrm{Az}=0.94)$ improved discrimination beyond that of SWD alone $(\mathrm{Az}=0.86)$. This suggests that future studies including vascularity as a discriminating factor may be strengthened by considering patient age or possibly just menopausal status.

Figure 5. Combined SWD-Age-GS index. The upper threshold line represents the discrimination obtained with equally weighted risks: sensitivity (true-positive fraction [TPF]), 95\%; and specificity (true-negative fraction [TNF]), 94\%. Requiring 100\% sensitivity did not alter the specificity in this case.

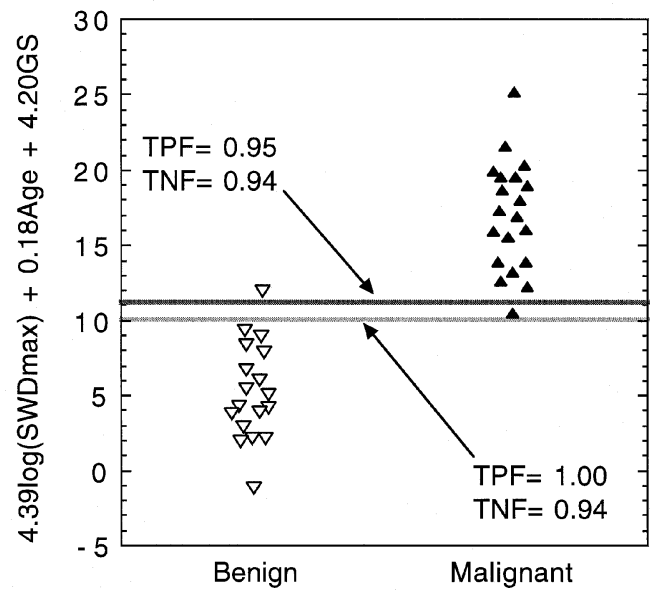




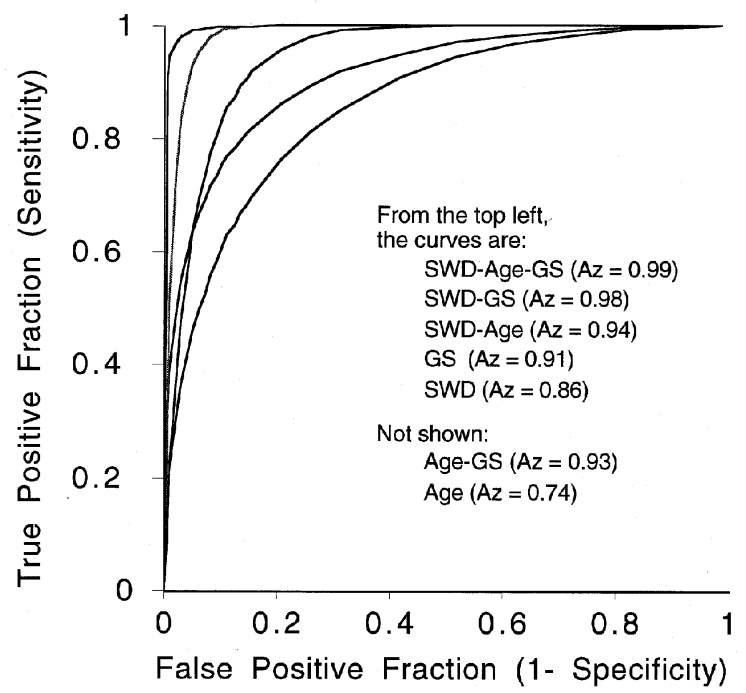

Figure 6. Receiver operating characteristic curves showing diagnostic performance $(\mathrm{Az})$ of SWD, GS, Age, and combinations thereof. Notice that the diagnostic accuracy of the GS-Age combination did not perform as well as the 2-variable combinations including vascularity, SWD-Age and SWD-GS. When all 3 variables were combined, SWD-Age-GS, the highest level of cancer discrimination was achieved.

On the basis of a criterion that a $2 \%$ or less chance of malignancy is required to avoid biop$\mathrm{sy}^{3}{ }^{3}$ the SWD-Age-GS technique described in this study could provide a useful discrimination tool. On the basis of a group of 38 patients, the findings suggest that by combining vascularity, age, and GS, the number of biopsies yielding benign results could be considerably reduced.

Table 2. Summary of Patient Diagnostics

\begin{tabular}{lc}
\hline Status & $\mathbf{n}$ \\
\hline Benign (average equivalent diameter, 1.1 cm)* & 18 \\
Resolved before biopsy & 1 \\
Benign breast tissue & 1 \\
Radial scar & 1 \\
Cyst & 2 \\
Fibrocystic changes & 6 \\
Fibroadenoma & 5 \\
Fibroadenoma with fibrocystic changes & 2 \\
Malignant (average equivalent diameter, $1.4 \mathrm{~cm})^{*}$ & 20 \\
Adenocarcinoma & 1 \\
Invasive ductal carcinoma & 6 \\
DCIS & 2 \\
Invasive ductal carcinoma with DCIS & 7 \\
Invasive LCIS & 1 \\
Invasive lobular carcinoma with LCIS & 1 \\
Invasive ductal carcinoma with LCIS & 1 \\
Other & 1 \\
\hline
\end{tabular}

DCIS indicates ductal carcinoma in situ; and LCIS, lobular carcinoma in situ. *Equivalent diameter is the diameter of a sphere whose volume is equivalent to the estimated volume of the mass.
Although vascularity has been presented as a potentially valuable measure in cancer discrimination of sonographic masses, its addition, possibly in combination with age, must provide better discrimination than age and GS to merit the addition of color flow to the ultrasonographic examination. A larger group of patients will be required to show with statistical significance that the combined index, SWD-GS-Age, offers consistently improved discrimination over the GS-Age index.

Hypothesizing that there exists a shell thickness limit within which most of the border vascularity information is contained, a 3-mm thickness was selected. Further consideration of this distance is warranted. Future work will include computing vascularity distributions as a function of distance from the mass border to assess the most representative shell thickness for the mass. The ROR and shell were each divided into 2 halves, proximal and distal, and SWD was computed for the proximal halves as well as the entire volume. Because shadowing by the mass is highly variable, the proximal ROR may be a more accurate representation of vascularity than the entire ROR.

Although our study describes a quantitative, and therefore objective, method for vascularity measurement, there are some limitations. Inherent in the speed computation is the assumption of isotropic blood flow. Specifically, the velocity as measured by the scanner was multiplied by a scaling factor of 2 to account for an isotropic distribution of Doppler angles. In addition the scans were performed with the transducer fixed perpendicular to the breast surface. Therefore, the angle of the transducer could not be rotated to find the best representation of each given blood vessel.

Table 3. Summary Regions of Interest Where Maximum SWD Occurred

\begin{tabular}{lr}
\hline Region & $\mathbf{n}$ \\
\hline Benign & 18 \\
Region 1 (entire ROR; Figure 2a) & 9 \\
Region 2 (proximal ROR; Figure 2b) & 0 \\
Region 3 (entire 3-mm shell; Figure 2c) & 2 \\
Region 4 (proximal 3-mm shell; Figure 2d) & 7 \\
Malignant & 20 \\
Region 1 (entire ROR; Figure 2a) & 2 \\
Region 2 (proximal ROR; Figure 2b) & 7 \\
Region 3 (entire 3-mm shell; Figure 2c) & 1 \\
Region 4 (proximal 3-mm shell; Figure 2d) & 10 \\
\hline
\end{tabular}


Table 4. Performance of Multivariable Measures When Optimized for Sensitivity

\begin{tabular}{lcc}
\hline Measure & Sensitivity, \% & Specificity, \% \\
\hline $\log S W D_{\max }-A g e-G S_{\text {avg }}$ & 100 & 94 \\
$\log S W D_{\max }-A g e$ & 100 & 72 \\
$\log S W D_{\max }-\mathrm{GS}_{\text {avg }}$ & 100 & 89 \\
Age-GS $_{\text {avg }}$ & 100 & 22 \\
\hline
\end{tabular}

The utility offered by these discrimination techniques relies on the objectivity of the vascularity measures. Identification of a mass and ROR delineation are the least objective steps in the vascularity analysis. The approach requires the radiologist to play a crucial role, but once the ROR is selected, all subsequent processing is done in software, implemented on commercial systems, and no adjustments are made as long as consistent scanner settings are used. Ultimately, discrimination is accomplished by combining SWD, a partially objective measure, with patient age and GS, a subjective measure.

Table 5. Statistical Significance of ROC Area (Accuracy) Comparisons

\begin{tabular}{llcl}
\hline Index 1 & Index 2 & Significance & $\boldsymbol{P}$ \\
\hline SWD-Age-GS & SWD & $\star$ & .013 \\
SWD-Age-GS & Age & $\dagger$ & .001 \\
SWD-Age-GS & GS & $\star$ & .024 \\
SWD-Age-GS & SWD-Age & $\neq$ & .086 \\
SWD-Age-GS & SWD-GS & NS & .230 \\
SWD-Age-GS & Age-GS & $\star$ & .037 \\
SWD-Age & SWD & $\neq$ & .090 \\
SWD-Age & Age & $\S$ & .010 \\
SWD-Age & GS & NS & .659 \\
SWD-Age & SWD-GS & NS & .336 \\
SWD-Age & Age-GS & NS & .871 \\
SWD-GS & SWD & $\star$ & .020 \\
SWD-GS & Age & $\S$ & .004 \\
SWD-GS & GS & $\star$ & .039 \\
SWD-GS & Age-GS & NS & .171 \\
Age-GS & SWD & NS & .395 \\
Age-GS & Age & $\S$ & .010 \\
Age-GS & GS & NS & .193 \\
SWD & Age & NS & .281 \\
SWD & GS & NS & .530 \\
Age & GS & $\ddagger$ & .065
\end{tabular}

NS indicates not significant.

${ }^{*} P \leq 0.05 ;+P \leq .001 ; \neq P \leq .1 ; \S P \leq .01$.

J Ultrasound Med 20:343-350, 2001

\section{Appendix}

The Bayesian type discriminator used to determine multivariable indices was based on standard methods derived in numerous texts. ${ }^{10,28}$ In our case, the simplifying assumption was made that both populations (benign and malignant) had normal distributions with equal covariance matrices, such that the decision "boundary" was a straight line, as mentioned in "Materials and Methods." The darker lines shown in Figures 3-5 represent the discrimination obtained with equally weighted risks (i.e., a likelihood ratio of 1 , $\ln [l(X)]=0)$. Thus, the particular discriminant function used, $h(X)$, is of the form ${ }^{10} h(X)=$ (M2 M1 $)^{\mathrm{T}} \Sigma^{-1} X+0.5\left(\mathrm{M1}^{\mathrm{T}} \Sigma^{-1} \mathrm{M} 1-\mathrm{M}^{\mathrm{T}} \Sigma^{-1} \mathrm{M} 2\right)$, where M1 and M2 are the expected vectors (or means), and $\sum$ represents both (equal) covariance matrices. For more information, see the related texts. ${ }^{10,28}$

\section{References}

1. Rosenberg RD, Hunt WC, Williamson MR, et al. Effects of age, breast density, ethnicity and estrogen replacement therapy on screening mammographic sensitivity and cancer stage at diagnosis. Radiology 1999; 209:511-518.

2. Sickles EA. Quality assurance. How to audit your own mammography practice. Radiol Clin North Am 1992; 30:265-275

3. Stavros AT, Thickman D, Rapp CL, et al. Solid breast nodules: use of sonography to distinguish benign from malignant lesion. Radiology 1995; 196:123-134.

4. Wilkens TH, Burke BJ, Cancelada DA, et al. Evaluation of palpable breast masses with color Doppler sonography and gray scale imaging. J Ultrasound Med 1998; 17:109-115.

5. Carson PL, Fowlkes JB, Roubidoux MA, et al. 3-D color Doppler image quantification of breast masses. Ultrasound Med Biol 1998; 24:945-952.

6. Fenn RC, Fowlkes JB, Moskalik A, et al. A handcontrolled 3-D ultrasound guide and measurement system. In: Lees S (ed). Acoustical Imaging. Vol 23. New York, NY: Plenum Press; 1997:237-242.

7. LeCarpentier GL, Bhatti PT, Fowlkes JB, et al. Utility of $3 \mathrm{D}$ ultrasound in the discrimination and detection of breast cancer. Radiological Society of North America Electronic Journal [serial online]; 1999. Available at: http://ej.rsna.org/ej3/0103-99.fin/ titlepage.html. 
8. Krücker JF, LeCarpentier GL. Meyer CR, et al. Examples of $3 D$ image registration for multimode, extended field of view, and sequential US imaging. Radiological Society of North America Electronic Journal [serial online]; 1999. Available at: http:// ej.rsna.org/ej3/0098-99.fin/titlepage.html.

9. Carson PL, Moskalik AP, Govil A, et al. 3D and 2D color flow display of breast masses. Ultrasound Med Biol 23:837-849, 1997.

10. Fukanaga K. Hypothesis testing. In: Rheinboldt W (ed). Introduction to Statistical Pattern Recognition. 2nd ed. New York, NY: Academic Press; 1990:51-58.

11. Metz CE. ROC Analysis Software. Chicago, IL: University of Chicago; 1997. Available at: http:// www-radiology.uchicago.edu/krl.

12. Swets JA, Pickett RM. Evaluation of Diagnostic Systems: Methods From Signal Detection Theory. New York, NY: Academic Press; 1982.

13. Minasian $\mathrm{H}$, Bamber JC. A preliminary assessment of an ultrasonic Doppler method for the study of blood flow in human breast cancer. Ultrasound Med Biol 1982; 8:357-364.

14. Kedar RP, Cosgrove DO, Bamber JC, et al. Automated quantification of color Doppler signals: a preliminary study in breast tumors. Radiology 1995; 197:39-43.

15. Madjar H, Prompeler HJ, Sauerbrei W, et al. Differential diagnosis of breast lesions by color Doppler. Ultrasound Obstet Gynecol 1995; 6:199-204.

16. Lee WJ, Chu JS, Huang CS, et al. Breast cancer vascularity: color Doppler sonography and histopathology study. Breast Cancer Res Treat 1996; 37:291-298.

17. Sahin-Akyar G, Sumer H. Color Doppler ultrasound and spectral analysis of tumor vessels in the differential diagnosis of solid breast masses. Invest Radiol 1996; 31:72-79.

18. Lee SK, Lee $T$, Lee $K R$, et al. Evaluation of breast tumors with color Doppler imaging: a comparison with image-directed Doppler ultrasound. J Clin Ultrasound 1995; 23:367-373.

19. Cosgrove DO, Kedar RD, Bamber JC, et al. Breast diseases: color Doppler US in differential diagnosis. Radiology 1993; 189:99-104.
20. Huber S, Delormes S, Knopp MV, et al. Breast tumors: computer assisted quantitative assessment with color Doppler US. Radiology 1994; 192:797-801.

21. Delorme S, Zuna I, Huber S, et al. Colour Doppler sonography in breast tumours: an update. Eur J Radiol 1998; 8:189-193.

22. Bell DS, Bamber JC, Eckersley RJ. Segmentation and analysis of colour Doppler images of tumour vasculature. Ultrasound Med Biol 1995; 21:635-647.

23. Folkman J, Merler E, Abernathy C, et al. Isolation of a tumor factor responsible for angiogenesis. J Exp Med 1971; 33:275-278.

24. Weidner N. Intratumor microvessel density as a prognostic factor in cancer. Am J Pathol 1995; 147:9-19.

25. Lee WJ, Chu JS, Houng SJ, et al. Breast cancer angiogenesis: a quantitative morphologic and Doppler imaging study. Ann Surg Oncol 1995; 2:246-251.

26. Buadu LD, Murkami J, Murayama S, et al. Colour Doppler sonography of breast masses: a multiparameter analysis. Clin Radiol 1997; 52:917-923.

27. Peters-Engl C, Medl M, Mirau M, et al. Color-coded and spectral Doppler flow in breast carcinomas: relationship with the tumor microvasculature. Breast Cancer Res Treat 1998; 47:83-89.

28. Afifi AA, Azen SP. Multivariate statistical methods. In: Statistical Analysis: A Computer Oriented Approach. New York, NY: Academic Press; 1979:288-318. 